\title{
Reliability of intrinsic birefringence estimated via the modified stress-optical rule
}

\author{
Yuki Okada, Osamu Urakawa and Tadashi Inoue
}

Intrinsic birefringence, a material constant for the orientational birefringence of polymers, has been measured by many researchers using a variety of methods. However, there is some disagreement among researchers regarding the reported values for the same polymer. This disagreement may be due to the complexity of conventional methods such as infrared dichroism and X-ray scattering methods to determine the degree of orientation. In addition, highly oriented polymers are often utilized, and the local structure of repeating units, including a side chain conformation, may be unnaturally changed owing to the high degree of orientation of the main chain. Alternatively, a new method using the modified stress-optical rule (MSOR) has been proposed for the measurement of intrinsic birefringence. In this article, the published data regarding the intrinsic birefringence for various polymers are assessed and the reliability of intrinsic birefringence estimated via MSOR is discussed.

Polymer Journal (2016) 48, 1073-1078; doi:10.1038/pj.2016.74; published online 21 September 2016

\section{INTRODUCTION}

Several polymeric materials have been widely used for optical devices, including optical discs, optical fibers and lenses. These particular materials are used because 'optical resins,' that is, polymeric materials for optical use, have features such as being light in weight, highly shock resistant and easy to process in comparison with inorganic glasses that are typical and traditional optical materials. However, optical resins have certain disadvantages, such as a lower heat resistance and a narrow range of refractive indices. In addition, polymeric materials are more birefringent under deformation than inorganic glasses. Birefringence induced by chain orientation is called 'orientational birefringence'. One material constant used to characterize the orientational birefringence is the intrinsic birefringence, $\Delta n_{0}$, that represents the virtual birefringence of perfectly oriented chains. $\Delta n_{0}$ can be related to the anisotropy of the polarizability of repeating units of the chain, $\Delta \alpha$, through the Lorentz-Lorenz equation.

$$
\Delta n_{0}=\frac{2}{9} \pi \frac{\left(\bar{n}^{2}+2\right)^{2}}{\bar{n}} \frac{\rho N_{\mathrm{A}}}{M_{0}} \Delta \alpha
$$

Here, $\bar{n}, \rho$ and $N_{\mathrm{A}}$ are the average refractive index, density and Avogadro's number, respectively. $M_{0}$ is the molar mass of the repeating units. Because $\bar{n}$ and $\rho$ do not vary much within polymer species, the intrinsic birefringence is determined mainly by $\Delta \alpha / M_{0}$. The value of $\Delta \alpha$ may be calculated by the valence-optical scheme, ${ }^{1-6}$ and therefore $\Delta n_{0}$ can be theoretically estimated from the molecular structure of repeating unit. In modern chemistry, $\Delta \alpha$ may be calculated by computational chemistry using software such as MOPAC. $^{7}$
For the case of uniaxially oriented polymers, the orientational birefringence, $\Delta n$, can be described with $\Delta n_{0}$ and the orientational degree, $f$.

$$
\begin{aligned}
& \Delta n=\Delta n_{0} f \\
& f=\frac{3\left\langle\cos ^{2} \theta\right\rangle-1}{2}
\end{aligned}
$$

Here, $\theta$ is the angle between the main chain direction of the polymers and the direction of uniaxial orientation; \langle\rangle represents the statistical average.

A fundamental experimental method to determine $\Delta n_{0}$ is the use of Equation (2) with the measurements of $\Delta n$ and $f$ for the uniaxially oriented samples, produced by stretching of polymers above the glass transition temperature. For measuring $f$, infrared dichroism or X-ray scattering have been utilized in most cases. However, measuring $f$ with these methods is somewhat complicated and time consuming; moreover, this may result in large experimental uncertainty. In addition, highly oriented polymers are often used for these measurements and the orientation of side groups of repeating units may be unnaturally changed by the high content of the trans conformation of the main chain. In any case, the reported values of $\Delta n_{0}$ sometimes vary with the researcher. Table 1 shows the typical $\Delta n_{0}$ values found in textbooks for some representative polymers ${ }^{8,9}$ (see Appendix for abbreviations of polymers). For example, the reported $\Delta n_{0}$ value for bisphenol A polycarbonate varies with the range of $0.1-0.2$. The large uncertainty suggests that the variation of $\Delta n_{0}$ values is not simply related to the statistical experimental error but rather to the systematic error related to the methods. 
Table 1 Intrinsic birefringence of representative polymers obtained from the literature

\begin{tabular}{lccccc}
\hline Sample & ${\text { Ref } A^{28}}^{28}$ & Ref $B^{29}$ & $\operatorname{Ref}^{30}$ & Ref $^{31}$ & Calculated $^{7}$ \\
\hline PS & -0.1 & -0.1 & & & -0.035 \\
PMMA & -0.004 & -0.001 & & & -0.008 \\
PVC & 0.01 & 0.0027 & & 0.0102 & -0.008 \\
PP & 0.04 & & $0.04-0.06$ & 0.035 & 0.0016 \\
PE & 0.052 & 0.044 & 0.12 & 0.058 & 0.005 \\
PA6 & 0.083 & & $0.08-0.1$ & 0.06 & \\
PC & 0.106 & 0.202 & 0.182 & & 0.0475 \\
PET & 0.22 & 0.105 & 0.220 .29 & & 0.0745 \\
PPE & 0.21 & & & & 0.0565 \\
PEEK & 0.34 & & & & \\
PVA & & & 0.0443 & 0.0433 & \\
PA610 & & 0.065 & & \\
Celu & & 0.055 & 0.086 & \\
P2VN & & & & -0.059 \\
PLLA & & & & 0.02 \\
PEN & & & & 0.118 \\
\hline
\end{tabular}

Abbreviations: Celu, cellulose; PA6, polyamide 6; PA610, polyamide 6-10; PC, bisphenol A polycarbonate; $\mathrm{PE}$, polyethylene; $\mathrm{PEEK}$, polyetheretherketone; $\mathrm{PEN}$, poly(ethylene naphthalate); PET, poly(ethylene terephthalate); PLLA, poly(L-lactic acid); PMMA, poly(methyl methacrylate); PP, atactic polypropylene; PPE, poly(phenyl ether); PS, polystyrene; PVA, poly(vinyl alcohol); PVC, poly(vinyl chloride); P2VN, poly(2-vinylnaphthalene).

Another material constant for the orientational birefringence is the stress-optical coefficient, $C_{\mathrm{R}}$. This coefficient is defined with the stress-optical rule (SOR) for polymer melt. ${ }^{10}$ The SOR states that the principal axes of the refractive index tensor and the stress tensor agree with each other and that the proportionality holds between the deviatoric parts of the refractive index and stress tensors. The proportionality coefficient, $C_{\mathrm{R}}$, can be related to the optical anisotropy of the segments, $\Delta \beta$. $^{11}$

$$
C_{\mathrm{R}}=\frac{2 \pi}{45 k_{\mathrm{B}} T} \frac{\left(\bar{n}^{2}+2\right)^{2}}{\bar{n}} \Delta \beta
$$

Here, $k_{\mathrm{B}}$ is the Boltzmann constant. The optical segment size, $n_{\mathrm{s}}$, can be defined as follows: ${ }^{6}$

$$
\Delta \beta \sim n_{\mathrm{s}} \Delta \alpha
$$

The optical segment size, $n_{\mathrm{s}}$, is determined by the chain statistics. For the first approximation, the number of repeating units per Kuhn segment, $n_{\mathrm{K}}$, can be used alternatively for $n_{\mathrm{s}}{ }^{12}$ The $n_{\mathrm{K}}$ values for various polymers have been determined. We can therefore estimate a value for $\Delta \alpha$ from the $C_{\mathrm{R}}$ data, and then $\Delta n_{0}$ can be calculated using Equation (1). Because measuring stress is convenient, this method has been widely used. In addition, a simple evaluation method of $C_{\mathrm{R}}$ has been proposed. ${ }^{13}$ However, theoretical considerations have revealed that the short-range ordering of chains because of the nematic interaction (NI) increases the $C_{\mathrm{R}}$ values for bulk systems. ${ }^{14-16}$ Although the strong NI effect has been realized for polyethylene (PE), evaluation of the NI effect for other polymers is very limited. For the case of bulk polystyrene, the NI effect is believed to give an $\sim 30 \%$ larger $C_{\mathrm{R}}$ value.

One problem of the estimation based on $C_{\mathrm{R}}$ measurements is that the orientational degree, $f$, is estimated from the stress that is sensitive to the intermolecular interaction such as the NI effect. An alternative method to estimate $\Delta n_{0}$ is using the modified stress-optical rule (MSOR) that is based on the simultaneous measurement of Young's modulus and the strain-optical coefficient. ${ }^{8}$ In this method, $f$ in the glassy state is estimated by using the pseudo-affine deformation model. In other words, $f$ is estimated from the macroscopic strain. The simple relation between $f$ and strain holds only in the glassy state because $f$ relaxes with time by molecular motions over longer time periods. To be more specific, the strain-induced birefringence in the glassy state has two origins: the orientational birefringence and the glassy birefringence. The details of the glassy birefringence are explained in the next section. To remove the glassy contribution, MSOR is applied with the simultaneously measured complex Young's modulus and the strainoptical coefficient. The merit of this method is its high applicability to various polymers, even those for which $n_{\mathrm{K}}$ data are not available. In this study, we compared $\Delta n_{0}$ data determined by various methods to identify the most reliable $\Delta n_{0}$ values. We note that all the data, including the results obtained via MSOR, were collected from published studies.

\section{PRINCIPLE}

\section{Estimation of the intrinsic birefringence through the MSOR}

Because the details of MSOR have been described elsewhere, ${ }^{8,17}$ we explain it only briefly here. For the case of the tensile deformation of rubbery materials, SOR holds well between their birefringence and the tensile stress. SOR is well established for various polymeric materials. ${ }^{10}$ SOR states that the molecular origin of birefringence and stress is the orientation of the segments. ${ }^{11}$ SOR is now a basic concept in various molecular theories to calculate the stress from the chain conformations. On the other hand, in the glassy state at low temperatures, the glassy response contributes to both the birefringence and the stress, in addition to the orientation of the segments. Consequently, the relationship between the birefringence and stress is rather complicated in the glassy state, and therefore MSOR becomes necessary. The MSOR for oscillatory tensile deformation can be written as follows: ${ }^{8}$

$$
\begin{aligned}
& E^{*}(\omega)=E_{\mathrm{R}}{ }^{*}(\omega)+E_{\mathrm{G}}{ }^{*}(\omega) \\
& O^{*}(\omega)=O_{\mathrm{R}}{ }^{*}(\omega)+O_{\mathrm{G}}{ }^{*}(\omega)=C_{\mathrm{R}} E_{\mathrm{R}}{ }^{*}(\omega)+C_{\mathrm{G}} E_{\mathrm{G}}{ }^{*}(\omega)
\end{aligned}
$$

Here, $E^{*}$ is the complex Young's modulus and $O^{*}$ is the complex strain-optical coefficient that is the complex ratio of oscillatory birefringence to oscillatory strain. $\omega$ is the angular frequency. The subscripts R and G represent the rubbery and the glassy components, respectively. MSOR states that the molecular origin of birefringence is the sum of the rubbery component and the glassy component. ${ }^{8,17}$ Regarding the two proportionality coefficients, $C_{\mathrm{R}}$ can be determined experimentally from the limiting values of $O^{\prime}(\omega) / E^{\prime}(\omega)\left(=O^{\prime \prime}(\omega) /\right.$ $\left.E^{\prime \prime}(\omega)\right)$ at $\omega \rightarrow 0$, and $C_{\mathrm{G}}$ can be determined from $O^{\prime \prime}(\omega) / E^{\prime \prime}(\omega)$ at $\omega \rightarrow \infty$. The component functions $E_{\mathrm{R}}{ }^{*}(\omega)$ and $E_{\mathrm{G}}{ }^{*}(\omega)$ are estimated by solving the simultaneous Equations (6) and (7). By applying the frequency-temperature superposition principle, the composite curve of $E_{\mathrm{R}}{ }^{*}(\omega)$ and $E_{\mathrm{G}}{ }^{*}(\omega)$ can be constructed. Details of the separation procedure and examples of $E_{\mathrm{R}}{ }^{*}(\omega)$ and $E_{\mathrm{G}}{ }^{*}(\omega)$ are described elsewhere. ${ }^{8,9}$ MSOR has been verified for more than 10 polymers. ${ }^{18}$ The separation into the rubbery and the glassy components makes it easier to interpret the viscoelastic properties of amorphous polymers around the glass-to-rubber transition zone. For example, Plazek ${ }^{19}$ reported that timetemperature superposition does not hold around the glass-to-rubber transition zone, and this can be attributed to a different temperature dependence of the $E_{\mathrm{R}}{ }^{*}(\omega)$ and $E_{\mathrm{G}}{ }^{*}(\omega) \cdot{ }^{8,20}$

The glassy component function is very similar to the complex modulus of oligomers. ${ }^{21}$ At high temperatures or at low frequencies, the glassy component is relaxed $\left(E_{\mathrm{G}^{\prime}}{ }^{\prime}(\omega), E_{\mathrm{G}}{ }^{\prime \prime}(\omega) \rightarrow 0\right)$ and Equations (6) and (7) are reduced to the ordinary SOR, $O^{*}(\omega)=C_{R} E_{R}{ }^{*}(\omega)=C_{R} E^{*}(\omega)$. Therefore, the rubbery component originates from the orientation of the segments. On the other hand, the molecular origin of the glassy component is assigned to the rotational orientation of repeating units about the main chain axis. ${ }^{17}$ The glassy component has strong nonlinearity and shows a large deformation yield. ${ }^{22}$ The birefringence of a highly stretched sample is mainly caused by the rubbery component. However, at small strains, the strain-induced birefringence includes both the contributions. In the glassy state, the limiting value of $C_{\mathrm{R}} E_{\mathrm{R}}{ }^{\prime}(\infty)$ at high frequencies $(\omega \rightarrow \infty)$ represents the birefringence induced by chain orientation without any relaxation. The value of $E_{\mathrm{R}}{ }^{\prime}(\infty)$ is obtained from 
Table 2 Stress-optical coefficient, characteristic modulus and $\Delta n_{0}$ via the MSOR method

\begin{tabular}{|c|c|c|c|c|c|c|c|}
\hline Polymer & $\mathrm{C}_{\mathrm{R}} / 10^{-12} \mathrm{~Pa}^{-1}$ & $E_{R}^{\prime} / M P a$ & $\Delta n_{0}$ & $C_{\mathrm{G}} / 10^{-12} \mathrm{~Pa}^{-1}$ & $E_{G^{\prime}} / M P a$ & $\mathrm{C}_{\mathrm{d}} / 10^{-12} \mathrm{~Pa}^{-1 \mathrm{a}}$ & $T_{\mathrm{r}} /{ }^{\circ} \mathrm{C}$ \\
\hline$\alpha-$ PMS $^{9}$ & -3400 & 16 & -0.091 & 20 & 2300 & -3.7 & 180 \\
\hline hvPB 17 & 790 & 20 & 0.026 & -9.5 & 1500 & 1 & -27 \\
\hline $\mathrm{vPB}^{17}$ & 200 & 30 & 0.01 & 13.5 & 1500 & 17.2 & -10 \\
\hline P4MS 32 & -6500 & 10.2 & -0.11 & 49 & 1820 & 12.5 & 120 \\
\hline PIP33 & 2000 & 35 & 0.117 & -10.7 & 2700 & 14 & -52 \\
\hline $\mathrm{APO}^{34}$ & 1720 & 13.4 & 0.038 & -13 & 1130 & 7.3 & 165 \\
\hline $\mathrm{PC}^{9}$ & 4700 & 26 & 0.203 & 35 & 1500 & 111 & 164 \\
\hline PCAP35 & 3300 & 21 & 0.116 & 27 & 1350 & 74 & 188 \\
\hline $\mathrm{PES}^{36}$ & 4300 & 26 & 0.186 & 40 & 1900 & 98 & 230 \\
\hline $\mathrm{PEI}^{36}$ & 8500 & 24 & 0.34 & 35 & 1700 & 153 & 222 \\
\hline PPE & 5000 & 20 & 0.17 & & & & \\
\hline PEN39 & 12400 & 16 & 0.34 & 63 & & 270 & 135 \\
\hline $\mathrm{PET}^{40}$ & 3800 & 26 & 0.165 & & & & \\
\hline
\end{tabular}

Abbreviations: APO, amorphous; hvPB, hydrogenated vinyl-polybutadiene; PArl, polyarylate from 2,2'-dicarboxybiphenyl and 2,2-bis(4-hydroxyphenyl) propane (bisphenol A); PC, bisphenol A polycarbonate; PCAP, bisphenol AP polycarbonate; PEI, poly(ether imide); PEN, poly(ethylene naphthalate); PES, poly(ether sulfone); PET, poly(ethylene terephthalate); PIP, polyisoprene; P4MS, poly(4-methyl styrene); $\alpha$-PMS, poly( $\alpha$-methyl styrene); PP, atactic polypropylene; PPE, poly(phenyl ether); PS, polystyrene; PSF, polysulfone; PtBS, poly(4-tert-butyl styrene); PVBPh, poly (vinylbiphenyl); UP, polyarylate from tere- and isophthalic acids (mole ratio is 1:1) and 2,2-bis(4-hydroxyphenyl)propane (bisphenol A); UPAP, polyarylate from tere- and isophthalic acids (mole ratio is 1:1) and 2,2,2-tris(4-hydroxyphenyl)phenylethane (bisphenol AP); MSOR, modified stress-optical rule.

aphoto-elastic coefficient, defined as the limiting value of $O^{\prime}(\omega) / E^{\prime}(\omega)$ at $\omega \rightarrow \infty$ in the glassy state.

the frequency dispersion of the composite curve of $E_{\mathrm{R}}{ }^{*}(\omega)$. Assuming the pseudo-affine deformation, the orientation degree, $f$, of repeating units can be estimated from the tensile strain, $\varepsilon$.

$$
f=\frac{3}{5} \varepsilon
$$

Considering Equation (2), we can estimate the intrinsic birefringence as

$$
\Delta n_{0}=\frac{5}{3} O_{\mathrm{R}}{ }^{\prime}(\infty)=\frac{5}{3} C_{\mathrm{R}} E_{\mathrm{R}}{ }^{\prime}(\infty)
$$

Thus, the intrinsic birefringence can be estimated from $C_{\mathrm{R}}$ and $E_{\mathrm{R}}{ }^{\prime}(\infty)$ data. We note this method is immune from the NI effect. The NI effect gives a larger $C_{\mathrm{R}}$ value but a smaller $E_{\mathrm{R}}{ }^{\prime}(\infty)$ value, resulting in the NI effect being canceled out for $C_{\mathrm{R}} E_{\mathrm{R}}{ }^{\prime}(\infty)$. In other words, a relatively weak coupling effect through the NI does not affect the orientation of repeating units in the glassy state. The NI effect is significant only in the rubbery zone. A detailed discussion of the NI effect has been described elsewhere. ${ }^{12}$

\section{Evaluation of the intrinsic birefringence from the stress-optical coefficient}

For a very few polymers, MSOR does not hold and therefore the separation into $E_{\mathrm{R}}{ }^{\prime}(\omega)$ and $E_{\mathrm{G}}{ }^{\prime}(\omega)$ cannot be performed. ${ }^{23}$ One of the reasons for this is the existence of the sub-relaxation around the glass transition zone. For such a case, we obtained $\Delta n_{0}$ values by a different method. From Equations (1), (4), and (5), we can derive the following equation.

$$
\Delta n_{0}=\frac{5}{3} C_{\mathrm{R}} \frac{3 \rho R T}{M_{\mathrm{K}}}
$$

This equation enables us to calculate $\Delta n_{0}$ values from the $C_{\mathrm{R}}$ and $M_{\mathrm{K}}$ data. ${ }^{12}$ The $\Delta n_{0}$ values thus determined are summarized in Table 3 . The NI effect can affect $\Delta n_{0}$ values in this method.
On the other hand, $E_{\mathrm{R}}{ }^{\prime}(\infty)$ can be generally related to the Rouse segment size, $M_{S}{ }^{8}$

$$
E_{\mathrm{R}}{ }^{\prime}(\infty)=\frac{3 \rho R T}{M_{\mathrm{S}}}
$$

Note that the $\Delta n_{0}$ values obtained via the SOR (Equation (10)) and MSOR methods (Equation (9)) agree for Equation (11) when $M_{\mathrm{K}}=M_{\mathrm{S}}$. In other words, the difference between the MSOR and the SOR methods can be attributed to the difference between $M_{\mathrm{K}}$ and $M_{\mathrm{S}}$. A previous study showed that the Rouse segment size is almost the same as the Kuhn segment size, $M_{\mathrm{K}}$, for undiluted systems. ${ }^{18}$ This means that either the SOR or MSOR method gives similar values for $\Delta n_{0}$, suggesting that the effect of NI is negligibly weak. On the other hand, the difference between the two segment sizes becomes significant in dilute solutions, where $M_{\mathrm{S}}$ becomes approximately 5 times larger than $M_{\mathrm{K}}$ owing to suppression of fast local dynamics. ${ }^{12,24-26}$

\section{RESULTS AND DISCUSSION}

\section{The intrinsic birefringence estimated via the MSOR method}

Table 2 summarizes $C_{\mathrm{R}}, E_{\mathrm{R}}{ }^{\prime}(\infty)$, and $\Delta n_{0}$ values for various polymers that have already been reported in previous studies. The $\Delta n_{0}$ value obtained via the MSOR method for polystyrene is -0.1 and agrees with the experimental value, indicating the potential of the MSOR method. As described before, MSOR does not hold for some polymers because of sub-relaxation in the glassy state. For these polymers, the intrinsic birefringence is estimated from the SOR method using the Kuhn segment size and $C_{\mathrm{R}}$ data. These results are given in Table 3.

Figure 1 compares the intrinsic birefringence between our results and the reported values. In Table 2, the data for 21 polymers are listed, and the $\Delta n_{0}$ has been measured for only 9 polymers by conventional methods. For polymers having several experimental values, all the data points are plotted in Figure 1. As described in the Introduction, the large scatter of the $\Delta n_{0}$ data suggests that the origin of the variation is not simply related to the statistical experimental errors. Therefore, we 
Table 3 Molar mass of the Kuhn segment, $M_{\mathrm{K}}$, and $\Delta n_{0}$ via the SOR method

\begin{tabular}{lcccc}
\hline Polymer & $M_{\mathrm{K}} / \mathrm{kg} \mathrm{mol}^{-1}$ & $\mathrm{C}_{\mathrm{R}} / 10^{-12} \mathrm{~Pa}^{-1}$ & $\Delta n_{0}$ & $T_{\mathrm{r}} /{ }^{\circ} \mathrm{C}$ \\
\hline $\mathrm{PS}^{8,41}$ & 0.76 & -5000 & -0.11 & 116 \\
$\mathrm{P}^{2} \mathrm{VN}^{42}$ & 1.39 & -8300 & -0.11 & 160 \\
$\mathrm{PP}^{17,41}$ & 0.19 & 1230 & $6.59 \times 10^{-2}$ & 2 \\
$\mathrm{PET}^{40}$ & 0.44 & 3800 & 0.18 & 100 \\
$\mathrm{PIB}^{41,43}$ & 0.27 & 1400 & $8.76 \times 10^{-2}$ & -45 \\
$\mathrm{PMMA}^{13,41}$ & 0.64 & 50 & $1.42 \times 10^{-3}$ & 150 \\
$\mathrm{PE}^{10,41}$ & 0.16 & 2100 & 0.22 & 150 \\
PVC $^{10,41}$ & 0.36 & -500 & $-2.39 \times 10^{-2}$ & 22 \\
PC $^{9}$ & 0.65 & 4700 & 0.16 & 164 \\
PPE $^{41}$ & 0.23 & 5000 & 0.37 & 100 \\
\hline
\end{tabular}

Abbreviations: PC, bisphenol A polycarbonate; PE, polyethylene; PET, poly(ethylene terephthalate); PIB, polyisobutylene; PMMA, poly(methyl methacrylate); PP, atactic polypropylene; PPE, poly(phenyl ether); PS, polystyrene; PVC, poly(vinyl chloride);

P2VN, poly(2-vinylnaphthalene); SOR, stress-optical rule.

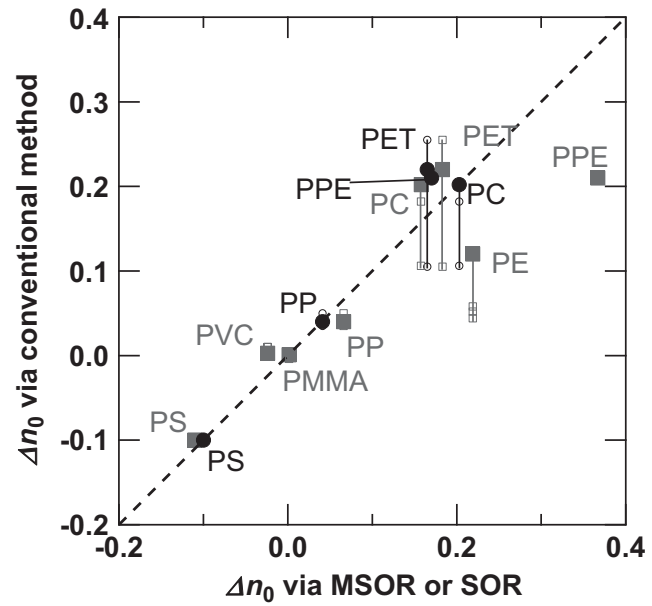

Figure 1 Comparison of $\Delta n_{0}$ via conventional methods and the modified stress-optical rule (MSOR) or stress-optical rule (SOR) method. See the text for details concerning the method determination. Black circles and red squares represent $\Delta n_{0}$ obtained via the MSOR and SOR methods, respectively. For polymers having several experimental values, the closest value to the data from the MSOR or SOR method is represented with filled marks and the others are represented with open marks. A full color version of this figure is available at Polymer Journal online.

did not calculate the average value. The elimination of the systematic errors originating from either the methods or the researchers because of the limited amount of data is a complicated issue, but here we found that some data points obtained via the conventional methods are close to the data obtained via the MSOR method. To illustrate this, the closest experimental value to the data obtained via the MSOR method in Figure 1 is represented by large filled marks. Figure 1 indicates a good agreement between our MSOR result and other selected experimental values.

For bisphenol A polycarbonate (PC),${ }^{9}$ two groups of the reported value $(\sim 0.1$ and $\sim 0.2)$ exist. According to MSOR, the intrinsic birefringence of PC would be $\sim 0.2$. The intrinsic birefringence of polymers containing benzene rings in their main chain, such as PET (poly(ethylene terephthalate)) and PPE (poly(phenyl ether)), is consistently $\sim 0.2$.

The results of the SOR method using $C_{\mathrm{R}}$ data and Kuhn segment size, which is shown by red squares, also give reliable estimations. As explained in the theory, the NI effect gives a larger $\Delta n_{0}$ value. A careful

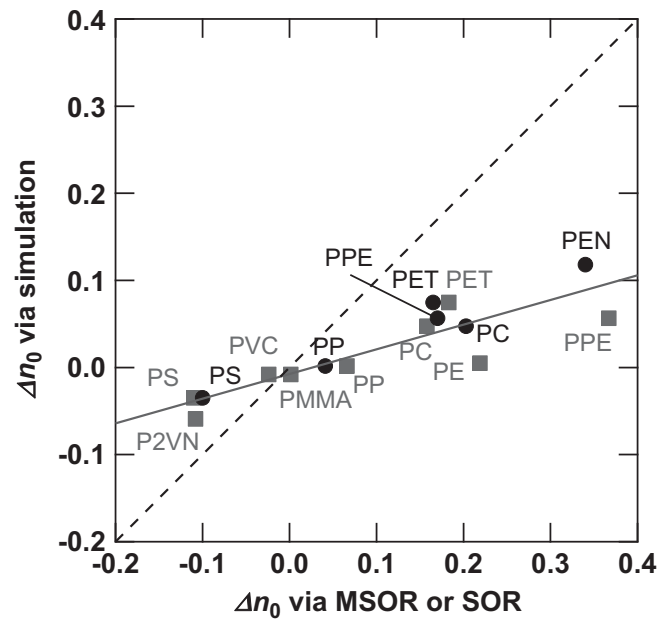

Figure 2 Comparison of $\Delta n_{0}$ by calculation and the modified stress-optical rule (MSOR) or stress-optical rule (SOR) method. See the text for details of the method determination. The blue line is an approximate straight line $\Delta n_{0}$ by each method. Black circles and red squares represent $\Delta n_{0}$ via the MSOR and SOR methods, respectively. A full color version of this figure is available at Polymer Journal online.

comparison of data from the SOR and MSOR methods reveals that this is true, except for PE, PC, and PPE. Note that polystyrene had negative $\Delta n_{0}$ and $\Delta n_{0}$ values estimated via the SOR method, located to the left side of those estimated by the MSOR method. A particularly large deviation was found for PE, and this can be attributed to the NI effect. The NI effect of PE was estimated to give approximately twice the values for $\Delta n_{0} \cdot{ }^{14}$ If we correct for the NI effect, $\Delta n_{0}$ obtained via the SOR method changes to $\sim 0.11$. The data point in Figure 1 is on the broken line by the horizontal shift. For the case of PPE, a large disagreement is observed. Because the value of $C_{R}$ for PPE is comparable to that for PC or PET, the reported value of $M_{\mathrm{K}}$ might include some experimental errors. For the case of PC, the SOR method provided a smaller value than MSOR method. The reason for this result is not clear at the present time. Strictly speaking, the relationship between $n_{\mathrm{S}}$ and $n_{\mathrm{K}}$ depends on details of chain statistics, and therefore the assumption, $n_{\mathrm{S}}=n_{\mathrm{K}}$ might be violated for PC. Further studies are needed for interpreting this irregular result.

For crystalline polymers such as PET, the $\Delta n_{0}$ value may be affected by the existence of crystals because in crystals, the local electric field is modified by regularly arranged neighboring chains. Rheo-optical measurements are normally performed with molten samples or quenched amorphous samples. The existence of crystals violates the SOR, hence making it detectable. ${ }^{27}$

\section{Comparison with simulations}

As described above, $\Delta \alpha$ values can be estimated by computer simulations. The reported values of $\Delta n_{0}$ obtained via a computational method are summarized in Table 1. For polymers having side groups, $\Delta \alpha$ varies with the conformation of the side groups. In this calculation, $\Delta \alpha$ and the rotational energy of the side groups were calculated by Quantum Chemical calculation (density functional theory: B3LYP/6$31+\mathrm{G}^{* *}$ by Gaussian 03) for various conformations of the side groups, and then the statistical average was obtained following the Boltzmann distribution. In a previous study, a comparison was made with a set of experimental values. ${ }^{7}$ However, for some polymers, the agreement was not good. Here, we compare their calculated values with the $\Delta n_{0}$ value obtained via the MSOR method in Figure 2. The proportional relationship holds well between the experimental and calculated 
values. Again, a large difference for $\mathrm{PE}$ can be attributed to the NI effect.

A careful observation reveals that a systematic deviation between the experimental and simulated values is observed. Their relationships are described by Equation (12)

$$
\Delta n_{0, \text { sim }}=A \Delta n_{0, \exp }+B
$$

Here, $\Delta n_{0, \text { sim }}$ and $\Delta n_{0, \exp }$ are the $\Delta n_{0}$ values obtained via the simulation and the experimental methods, respectively. The values of $A$ and $B$ are constant and they are 0.28 and -0.075 , respectively. We have not identified the origin of this systematic deviation, particularly why $A \neq 1$. Regarding the non-zero $B$ parameter, we note that the Lorentz-Lorenz equation (Equation (1)) considers only the orientation of anisotropic repeating units. However, an anisotropic internal electrostatic field may be created by the surrounding anisotropic chains, and this can contribute to the birefringence. To consider such an effect, let us consider perfectly oriented chains. The free volume of polymeric materials is typically $\sim 0.025$ around the glass transition region, and its spatial distribution would also be anisotropic because the chain itself is rod-like and anisotropic. For such a system, we should consider the form birefringence of the free volume or chains. A very preliminary calculation predicts a form birefringence of $\sim 0.01$. The non-ideal term that is not considered in the Lorentz-Lorenz equation is an important issue for future work.

\section{CONCLUSION}

We have discussed the reliability of intrinsic birefringence estimated via the MSOR and the SOR methods. The good agreement between our results and other experimental values has been established. On the other hand, a systematic deviation between the experimental values and the simulation values has been observed. An improvement of the simulation method is considered as an important issue for the precise prediction of intrinsic birefringence in addition to the consideration of the form birefringence of the chain.

\section{CONFLICT OF INTEREST}

The authors declare no conflict of interest.

\section{ACKNOWLEDGEMENTS}

This research was partially supported by a Grant-In-Aid (16H04204) from the ministry of Education, Culture, Sports, Science and Technology, Japan.

1 Nagai, K. Stress-optical coefficient of polymeric networks. Stereo-irregular and multirepeat polymers. J. Chem. Phys. 47, 2052 (1967).

2 Nagai, K. Stress-optical coefficient of polyethylene networks. J. Chem. Phys. 49, 4212 (1968).

3 Ishikawa, T. \& Nagai, K. Stress-optical coefficient of cis-1,4-polybutadiene and cis-1, 4-polyisoprene networks. Measurements on cis-1,4-polybutadiene networks and theoretical interpretation. J. Polym. Sci. A2 7, 1123 (1969).

4 Nagai, K. Stress-optical coefficient of polymeric networks. Stereo-irregular and multirepeat polymers. J. Chem. Phys. 51, 1265 (1969).

5 Ishikawa, T. \& Nagai, K. Stress-optical coefficient of trans-1,4-polybutadiene and trans1,4-polyisoprene networks. Measurement and theoretical interpretation. Polym. J. 1, 116 (1970).

6 Volkenstein, M. V. Configurational Statics of Polymeric Chains Interscience (1963).

7 Tanaka, T., Aoyagi, T. \& Shirai, H. In Polymer Processing Society 22nd Annual Meeting, Proceeding (2006)

8 Inoue, T., Okamoto, H. \& Osaki, K. Birefringence of amorphous polymers .1. Dynamic measurement on polystyrene. Macromolecules 24, 5670-5675 (1991).
9 Inoue, T., Hwang, E. J. \& Osaki, K. Birefringence of amorphous polymers .5. Dynamic measurements on poly(alpha-methylstyrene) and polycarbonate. J. Rheol. 36 1737-1755 (1992).

10 Janeschitz-Kriegl, H. Polymer Melt Rheology and Flow Birefringence (Springer-Verlag, Berlin, 1983).

11 Doi, M. \& Edwards, S. F. The Theory of Polymer Dynamics (Clarendon Press, Oxford, 1986).

12 Inoue, T. On the relationship between viscoelastic segments and Kuhn segments; Strain-induced chain orientation in fast deformation. Macromolecules 39, 4615-4618 (2006).

13 Ryu, D. S., Inoue, T. \& Osaki, K. A simple evaluation of stress-optical coefficient of polymers. Nihon Reoroji Gakk. 24, 129-132 (1996).

14 Tanaka, T. \& Allen, G. Short-range order in deformed polymer networks. Macromolecules 10, 426-430 (1977).

15 Doi, M., Pearson, D., Kornfield, J. \& Fuller, G. Effect of nematic interaction in the orientational relaxation of polymer melts. Macromolecules 22, 1488-1490 (1989).

16 Doi, M. \& Watanabe, H. Effect of nematic interaction on the Rouse dynamics. Macromolecules 24, 740-744 (1991).

17 Inoue, T., Mizukami, Y., Okamoto, H., Matsui, H., Watanabe, H., Kanaya, T. \& Osaki, K. Dynamic birefringence of vinyl polymers. Macromolecules 29, 6240-6245 (1996).

18 Inoue, T. \& Osaki, K. Role of polymer chain flexibility on the viscoelasticity of amorphous polymers around the glass transition zone. Macromolecules 29, 1595-1599 (1996).

19 Plazek, D. J. Temperature dependence of viscoelastic behavior of polystyrene. J Phys Chem. 69, 3480 (1965).

20 Inoue, T., Onogi, T., Yao, M. L. \& Osaki, K. Viscoelasticity of low molecular weight polystyrene. Separation of rubbery and glassy components. J. Polym. Sci. Pol. Phys. 37, 389-397 (1999).

21 Inoue, T., Onogi, T. \& Osaki, K. Dynamic birefringence of oligostyrene: a symptom of "polymeric" mode. J. Polym. Sci. Pol. Phys. 38, 954-964 (2000).

22 Inoue, T., Ryu, D. S. \& Osaki, K. A rheo-optical study on polystyrene under large tensile deformation around the glass transition temperature. Macromolecules 31, 6977-6983 (1998).

23 Osaki, K., Okamoto, H., Inoue, T. \& Hwang, E. J. Molecular interpretation of dynamic birefringence and viscoelasticity of amorphous polymers. Macromolecules 28 3625-3630 (1995).

24 Peterson, S. C., Echeverria, I., Hahn, S. F., Strand, D. A. \& Schrag, J. L. Apparent relaxation-time spectrum cutoff in dilute polymer solutions: an effect of solvent dynamics. J. Polym. Sci. Pol. Phys. 39, 2860-2873 (2001).

25 Inoue, T., Uematsu, T. \& Osaki, K. The significance of the Rouse segment: its concentration dependence. Macromolecules 35, 820-826 (2002).

26 Larson, R. G. An explanation for the high-frequency elastic response of dilute polymer solutions. Macromolecules 37, 5110-5114 (2004).

27 Ryu, D. S., Inoue, T. \& Osaki, K. A Rheo-optical study of polymer crystallization in the process of elongation of films. Polymer 39, 2515-2520 (1998).

28 Oji Scientific Instruments. Birefringence and orientation function, technical report, https://www.oji-keisoku.co.jp/products/kobra/img/gijutu42.pdf. (2012).

29 Ide, F. in Refractive Index Control of Clear Polymers 3-16 (Academic Press Center, Tokyo, Japan, 1998).

30 Fuji, M., Sakurai, K. \& Fujita, A. in Refractive Index Control of Clear Polymers 122-132 (Academic Press Center, Tokyo, Japan, 1998).

31 Okamura, S. Introduction of Polymer Chemistry (Kagakudojin, Kyoto, Japan, 1981).

32 Inoue, T., Matsui, H. \& Osaki, K. Molecular origin of viscoelasticity and chain orientation of glassy polymers. Rheol. Acta. 36, 239-244 (1997).

33 Okamoto, H., Inoue, T. \& Osaki, K. Birefringence of amorphous polymers. 8. Viscoelasticity and birefringence of polyisoprene. J. Polym. Sci. Pol. Phys. 33, 417-424 (1995).

34 Inoue, T., Takiguchi, O., Osaki, K., Kohara, T. \& Natsuume, T. Dynamic birefringence of amorphous polyolefins. 1. Measurements on poly[1-ethyl-5-methyl-octahydro-4,7methano-1h-Indene-1(2),3-Diyl]. Polym. J. 26, 133-139 (1994).

35 Inoue, T., Hwang, E. J. \& Osaki, K. Dynamic birefringence of Bispheol-AP Polycarbonate. Bull. Inst. Chem. Res. 70, 161-168 (1992).

36 Hwang, E. J., Inoue, T. \& Osaki, K. Viscoelasticity of some engineering plastics analyzed with the modified stress-optical rule. Polym. Eng. Sci. 34, 135-140 (1994).

37 Inoue, T., Hwang, E. J. \& Osaki, K. Birefringence of amorphous polyarylates. 2. Dynamic measurement on a polyarylate with low optical anisotropy. Polymer 38, 1029-1034 (1997).

38 Osaki, K., Inoue, T., Hwang, E. J., Okamoto, H. \& Takiguchi, O. Dynamic birefringence of amorphous polymers. J. Non-Cryst. Solids 172, 838-849 (1994).

39 Inoue, T., Matsui, H., Murakami, S., Kohjiya, S. \& Osaki, K. Strain-induced birefringence and molecular structure of glassy polymers. Polymer 38, 1215-1220 (1997).

40 Ryu, D. S., Inoue, T. \& Osaki, K. A birefringence study of polymer crystallization in the process of elongation of films. Polymer 39, 2515-2520 (1998).

41 Brandrup, J. Polymer Handbook (John Wiley and Sons, Inc, New York, NY, USA, 1975)

42 Hwang, E. J., Inoue, T., Osaki, K. \& Takano, A. Viscoelasticity and birefringence of poly (2-vinylnaphthalene). Nihon Reoroji Gakk. 22, 129-134 (1994).

43 Okamoto, H., Inoue, T. \& Osaki, K. Viscoelasticity and birefringence of polyisobutylene. J. Polym. Sci. Pol. Phys. 33, 1409-1416 (1995). 


\section{APPENDIX}

\section{Abbreviations Used for Polymer Identification}

\begin{tabular}{|c|c|}
\hline$\alpha$-PMS & $\operatorname{poly}(\alpha$-methyl styrene $)$ \\
\hline APO & $\begin{array}{l}\text { amorphous polyolefin, poly[1-ethyl-5-methyl-octahy- } \\
\left.\text { dro- } 4,7 \text {-methano- } 1 H \text {-indene- } 1^{2}, 3 \text {-diyl }\right]\end{array}$ \\
\hline $\mathrm{PP}$ & atactic polypropylene \\
\hline hvPB & hydrogenated vinyl-polybutadiene \\
\hline vPB & vinyl-polybutadiene \\
\hline UP & $\begin{array}{l}\text { polyarylate from tere- and isophthalic acids (mole ratio is } \\
\text { 1:1) and 2,2-bis (4-hydroxyphenyl)propane (bisphenol A) }\end{array}$ \\
\hline UPAP & $\begin{array}{l}\text { polyarylate from tere- and isophthalic acids (mole ratio } \\
\text { is } 1: 1 \text { ) and 2,2,2-tris(4-hydroxyphenyl)phenylethane } \\
\text { (bisphenol AP) }\end{array}$ \\
\hline PtBS & poly(4-tert-butyl styrene) \\
\hline PCAP & bisphenol AP polycarbonate \\
\hline PEI & poly(ether imide) \\
\hline PES & poly(ether sulfone) \\
\hline PEN & poly(ethylene naphthalate) \\
\hline PET & poly(ethylene terephthalate) \\
\hline PES & poly(ethylene sulfone) \\
\hline PIB & polyisobutylene \\
\hline PIP & polyisoprene \\
\hline
\end{tabular}

PMMA poly(methyl methacrylate)

P4MS poly(4-methyl styrene)

PPE poly(phenyl ether)

PS polystyrene

PSF polysulfone

PVC poly(vinyl chloride)

PE polyethylene

PA6 polyamide 6

PA610 polyamide 6-10

PC bisphenol A polycarbonate

PEEK polyetheretherketone

PVA poly(vinyl alcohol)

Celu cellulose

P2VN poly(2-vinylnaphthalene)

PLLA poly(L-lactic acid)

P4MS poly(4-methylstyrene)

PVBPh poly(vinylbiphenyl)

PArl polyarylate from 2,2'-dicarboxybiphenyl and 2,2-bis (4-hydroxyphenyl) propane (bisphenol A) 